\title{
Gossypiboma in a post caesarean patient: surgeon's dilemma
}

\author{
Sonia Balbir Singh, Hemali H. Sinha*, Sangam Jha
}

Department of Obstetrics and Gynaecology, AIIMS, Patna, Bihar, India

Received: 15 September 2020

Accepted: 28 October 2020

\section{*Correspondence:}

Dr. Hemali Heidi Sinha,

E-mail: drhemalihs@aiimspatna.org

Copyright: () the author(s), publisher and licensee Medip Academy. This is an open-access article distributed under the terms of the Creative Commons Attribution Non-Commercial License, which permits unrestricted non-commercial use, distribution, and reproduction in any medium, provided the original work is properly cited.

\begin{abstract}
Gossypiboma or retained surgical sponge is a relatively common, but underreported event due to medico legal implications and the exact incidence in not known. The clinical presentation and the time of presentation is variable because of which the diagnosis may be challenging. A high degree of suspicion is required to reach the diagnosis. Gossypiboma can have catastrophic implications for patients and healthcare professionals. We present a case of a 23 year old patient with retained surgical mop after caesarean section, who came with complaints of fever and pain abdomen. The purpose of presenting this case is to sensitize the surgeons to always keep retained surgical items as their differential diagnosis in cases of post-operative abdominal pain or fever, to decrease the time between surgery and diagnosis so as to minimize the morbidity to patient. The World health organization (WHO) surgical safety checklist should be strictly adhered to.
\end{abstract}

Keywords: Gossypiboma, Caesarean section, WHO surgical safety checklist

\section{INTRODUCTION}

Gossypiboma is a sponge or a swab retained involuntarily within the body during a surgical procedure. The incidence of retained surgical items is difficult to estimate due to under-reporting of cases due to medico legal implications. The reported incidence varies from 1 per 3000 to 5000 procedures. ${ }^{1}$ The wide variation in the incidence depends on the type of procedure, operation theatre personnel, experience of the surgeon, frequency of reporting of adverse incidents and hospital policies. The diagnosis is challenging because of variation in clinical and imaging presentation depending on time elapsed after surgery. Ultrasonography (USG), computerized tomography (CT) and magnetic resonance imaging are usually used as important diagnostic tools. The incidence of this catastrophic complication can be significantly reduced by strictly adhering to World health organization (WHO) surgical safety checklist. ${ }^{2}$ Awareness among surgeons and radiologists can lead to early diagnosis and intervention, preventing further complications.

\section{CASE REPORT}

A 23 years old P1L1 female with history of gestational hypertension presented on day 18 post Lower segment cesarean section (LSCS) with complaints of fever and generalized pain abdomen for 10 days. She had delivered a healthy $2.7 \mathrm{~kg}$, male baby, at 36 weeks period of gestation via emergency LSCS in view of premature rupture of membranes (PROM) with gestational hypertension, 18 days back. One blood transfusion was done post LSCS. Patient was kept at that hospital for 8 days post LSCS and was discharged thereafter with no complaints.

The day after discharge, she developed fever with chills and rigor followed by pain abdomen and abdominal distension for which outpatient management was done. Thereafter, patient went to another hospital for same, she was admitted and was managed conservatively. As the symptoms were not subsiding, she was referred to our hospital for further management. On examination- patient had high grade fever, with moderate degree of anemia with 
pedal edema. Abdomen was distended, generalized tenderness was present and the uterus could not be palpated. On pelvic examination, the cervical os was closed, size of the uterus could not be appreciated due to tenderness and lochia was healthy. On investigationhemoglobin was 8.9 gm\% with normal leucocyte count and platelet count- 6,65,000. Ultrasonography showed peritonitis with large multiple loculated collections in the perihepatic, perisplenic space, paracolic gutter and the pelvis; with the largest collection measuring approximately $500 \mathrm{cc}$ on the left side for which a pigtail catheter was inserted and the pus was drained, by the sonologist.

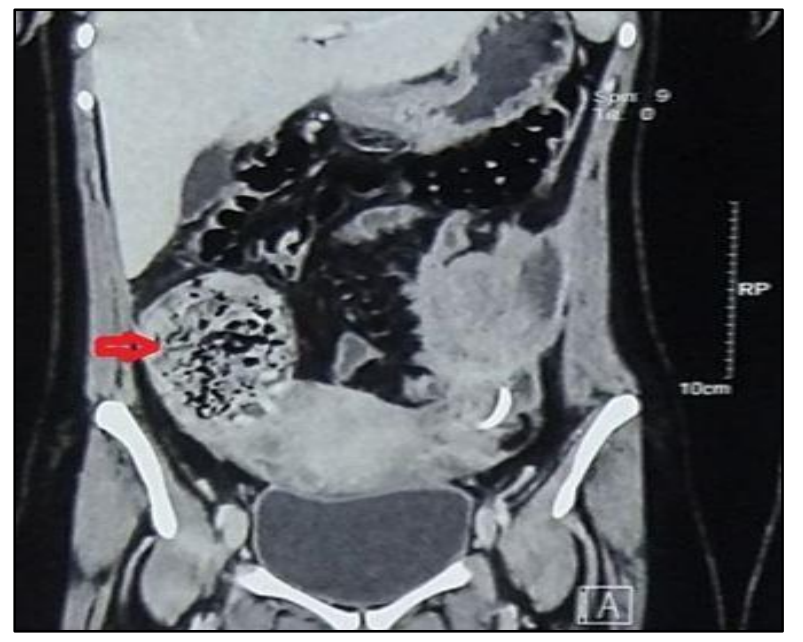

Figure 1: Gossypiboma seen on CT scan film (coronal section).

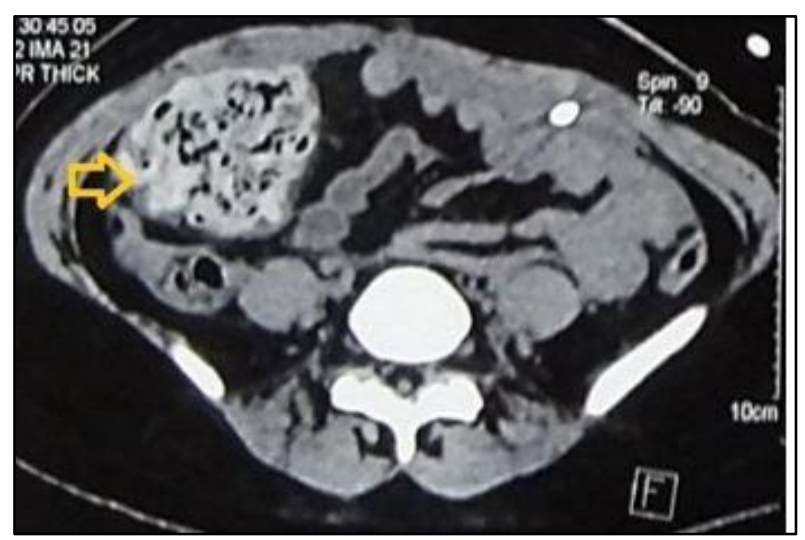

Figure 2: Gossypiboma seen on CT scan film (transverse section).

During this conservative treatment, after draining approximately $300 \mathrm{ml}$ of pus, clinically a $4-5 \mathrm{~cm}$ swelling was felt in right iliac fossa for which USG was done which showed a thick septate collection in the same spaces with near complete resolution in the paracolic gutter. At the site of clinical swelling, posterior acoustic shadow was seen but the region could not be evaluated on USG and contrastenhanced computed tomography (CECT) was suggested.
CECT (figure1, 2) revealed a well-defined sharply outlined mass at right lower quadrant of abdomen with hypodense area containing multiple air foci suggestive of Gossypiboma. There was evidence of communication of collection from endometrial cavity into right intraperitoneal collection.

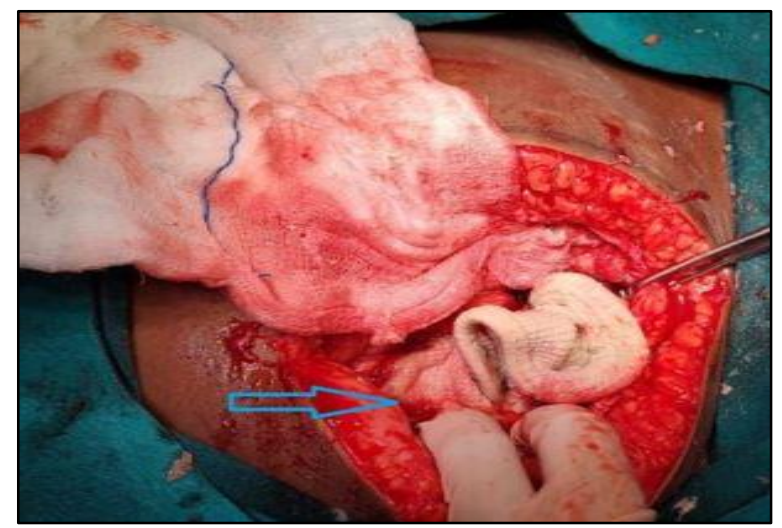

Figure 3: Gossypiboma in situ seen during laparotomy (blue arrow).

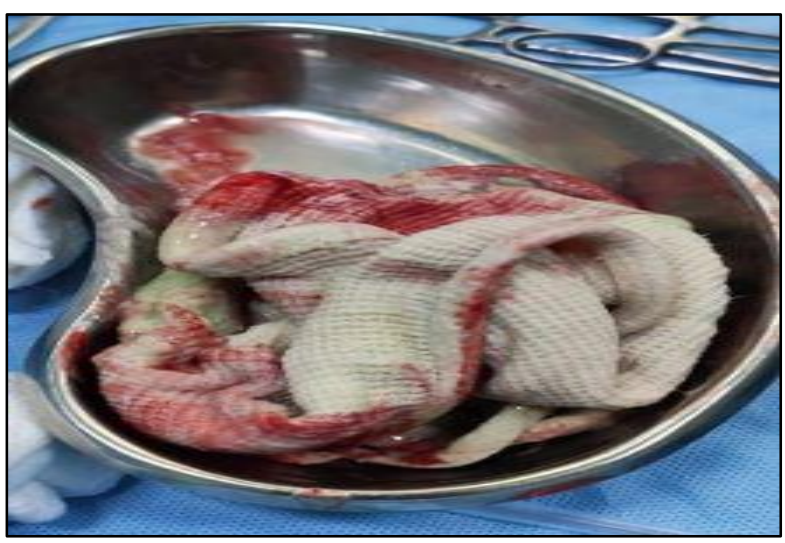

Figure 4: Gossypiboma retrieved.

Decision for immediate laparotomy was taken. Dense adhesions were present between layers of anterior abdominal wall; bowel loops were adhered to each other and to the anterior and lateral abdominal wall. Uterus was involuted and adhered densely with bladder and omentum anteriorly. Frank pus was present in abdomen. Right paracolic gutter was reached after adhesiolysis; an organized mass (mop covered with pus and omentum) was present which was removed (figure 3, 4). Necrotic omentum, present below the umbilicus and right subcostal region was removed. Post-operative period was uneventful, and patient was discharged on post-operative day 8 after stitch removal.

\section{DISCUSSION}

A retained surgical sponge or gossypiboma is an underreported complication occurring most commonly after abdominal surgeries. The clinical appearance of gossypiboma can vary from being asymptomatic to vague 
and varied symptoms like fever, abdominal pain, distension, nausea, vomiting, discharge from stitch site, weight loss, palpable mass. Retained surgical items can cause two different types of tissue reaction; first is exudative reaction which presents early in the postoperative period, and the second is aseptic fibrous reaction, which is slow and can remain asymptomatic for years. ${ }^{3}$ Stawicki et al showed that the most common clinical and diagnostic findings were focal pain, fluid or abscess collection or mass, and the most common pathological findings were exudative reaction, fibrosis, purulence, or abscess. ${ }^{4}$ CT scan is the first-choice diagnostic imaging technique for excluding gossypibomas because of its higher sensitivity, which shows typical spongiform pattern or heterogeneous central areas due to gas, calcification, and radiopaque markers. ${ }^{5,6}$ Ultrasound can also be used as a diagnostic tool which shows a poorly defined echogenic area with intense posterior acoustic shadowing. ${ }^{7}$

Prevention is always better than cure and that too in a scenario where surgical removal is the cure. WHO surgical safety checklist should be used to reduce reliance on memory. Counting mops and instruments before operation, just before closing abdomen and after the operation should be made a routine. The avoidance of conditions leading to human error like, fear, anxiety, anger, time pressure, interruptions; avoidance of nursing staff change in between the operation decrease such mishaps. In case of necessity of nursing staff change in between the surgery due to unavoidable circumstances, proper handover about the counts should be given. Computer-assisted system can be used for counting sponges using barcodes, which can detect significantly more counting discrepancies compared to traditional counting protocols and can further decrease the incidence of such events. ${ }^{8}$

\section{CONCLUSION}

WHO surgical safety checklist should be strictly adhered to. In case of discrepancy in count, appropriate action should be taken immediately to decrease morbidity of the patient. In case, patient presents with post-operative complications like fever with abdominal pain or discharge from wound, a high index of suspicion for retained sponge should be considered.
Funding: No funding sources Conflict of interest: None declared Ethical approval: Not required

\section{REFERENCES}

1. Kiernan F, Joyce M, Byrnes CK, O'Grady H, Keane FB, Neary P. Gossypiboma: a case report and review of the literature. Ir J Med Sci. 2008;177(4):389-91.

2. Goldberg JL, Feldman DL. Implementing AORN recommended practices for prevention of retained Surgical Items. AORN J. 2012;95:205-19.

3. Hariharan D, Lobo DN. Retained surgical sponges, needles and instruments. Ann R Coll Surg Engl. 2013;95:87-92.

4. Stawicki SP, Cook CH, Anderson HL 3rd, Chowayou L, Cipolla J, Ahmed HM, et al. Natural history of retained surgical items supports the need for team training, early recognition, and prompt retrieval. Am J Surg. 2014;208:65-72.

5. Yildirim T, Parlakgumus A and Yildirim S. Diagnosis and management of retained foreign objects. J Coll Physicians Surg Pak. 2015;25:367-71.

6. Lu YY, Cheung YC, Ko SF, Ng SH. Calcified reticulate rind sign: a characteristic feature of gossypiboma on computed tomography. World $\mathbf{J}$ Gastroenterol. 2005;11:4927-9.

7. Kumar GVS, Ramani S, Mahajan A, Jain N, Sequeira $\mathrm{R}$, Thakur M. Imaging of retained surgical items: A pictorial review including new innovations. Indian $\mathbf{J}$ Radiol Imaging. 2017;27(3):354-61.

8. Greenberg CC, Diaz-Flores R, Lipsitz SR, Regenbogen SE, Mulholland L, Mearn F, et al. Barcoding surgical sponges to improve safety: a randomized controlled trial. Ann Surg. 2008;247:6126.

Cite this article as: Singh $\mathrm{SB}$, Sinha $\mathrm{HH}$, Jha S. Gossypiboma in a post caesarean patient: surgeon's dilemma. Int J Reprod Contracept Obstet Gynecol 2020;9:5137-9. 